\title{
Treatment Patterns and Associated Health Care Costs Before and After Treatment Initiation Among Pulmonary Arterial Hypertension Patients in the United States
}

\author{
Charles D. Burger, MD, FCCP; A. Burak Ozbay, MBA, PhD; Howard M. Lazarus, MD; Ellen Riehle, MPH; \\ Leslie B. Montejano, MA, CCRP; Gregory Lenhart, MS; and R. James White, MD, PhD
}

\begin{abstract}
BACKGROUND: Despite multiple treatment options, the prognosis of pulmonary arterial hypertension (PAH) remains poor. PAH patients experience a high economic burden due to comorbidities, hospitalizations, and medication costs. Although combination therapy has been shown to reduce hospitalizations, the relationship between treatment, health care utilization, and costs remains unclear.
\end{abstract}

OBJECTIVE: To provide a characterization of health care utilization and costs in real-world settings by comparing periods before and after initiating PAH-specific treatment.

METHODS: This retrospective study identified PAH patients in the Truven Health MarketScan Commercial and Medicare Supplemental Databases between 2010 and 2014 who initiated treatment with endothelin receptor antagonists (ERAs), phosphodiesterase- 5 inhibitors (PDE-5Is), or soluble guanylate cyclase ( $\mathrm{GGC}$ ) stimulators. The index date was the date of the first PAH pharmacy claim. We included patients with $\geq 2$ medical claims with diagnoses for PAH (ICD-9-CM: 416.0, 416.8) or PAH-related conditions and continuous enrollment in medical and pharmacy benefits for the 6 months before and after the index date. Treatment patterns were assessed at the drug class level (ERAs, PDE-5Is, sGC stimulators, and prostacyclins) from outpatient pharmacy claims during the 6-month post-index period. All-cause and PAH-related utilization and costs were measured. McNemar's and paired t-tests were used to compare patients' health care resource utilization and costs in the 6-month pre- and posttreatment periods.

RESULTS: A total of 3,908 patients met the selection criteria. The study sample was $63 \%$ female with a mean age of $63 \pm 15$ years. Only $5 \%$ of patients began initial combination therapy for PAH, defined as claims for $\geq 2$ medication classes within the first 30 days of treatment. Treatment interruption ( $\geq 30$-day gap in days supply) of any PAH-specific medication was observed in $38 \%$ of patients. Compared with the 6 -month pre-index period, the proportion of patients in the 6-month post-index period with any inpatient admission decreased, $42 \%$ versus $30 \%(P<0.001)$. In addition, PAH-related inpatient admissions decreased in the 6-month post-index period from $7 \%$ to $3 \%(P<0.001)$. After treatment initiation, patients' nonpharmacy medical costs decreased from $\$ 48,200(S D=\$ 117,686)$ to $\$ 33,962$ ( $S D=\$ 90,294 ; P<0.001)$, mainly attributable to reduced inpatient costs. However, total average medical costs including pharmacy costs remained comparable after treatment initiation (pre-index period $=\$ 51,455$ vs. post-index period $=\$ 53,923 ; P=0.213$ ).

CONCLUSIONS: This study found that while patients' PAH-related pharmacy costs increased after treatment initiation, the increase was offset by reduced inpatient utilization; therefore, total health care costs remained constant. While the majority of patients in this study were treated with monotherapy, the recently completed AMBITION study indicated that initial combination therapy with ambrisentan plus tadalafil reduced PAH-related hospitalizations compared with initial monotherapy with either of these agents. Future cost analyses of patients treated with combination therapy will be required to determine the economic effect of initial combination therapy.

J Manag Care Spec Pharm. 2018;24(8):834-42

Copyright $\odot 2018$, Academy of Managed Care Pharmacy. All rights reserved.

\section{What is already known about this subject}

Pharmacy costs for pulmonary arterial hypertension (PAH) medications are high; however, previous studies have shown significant reductions in overall costs following treatment initiation due to reduced health care utilization.

Hospitalizations are common and contribute substantially to the PAH cost burden.

Recommendations for PAH treatment have evolved with the recent evidence in support of initial combination therapy.

\section{What this study adds}

This study provides observational descriptive evidence that total health care spending remained unchanged during the first 6 months following PAH treatment initiation due to the offsetting factors of increased PAH-related pharmacy costs and decreased inpatient utilization.

Treatment pattern analyses in a large representative U.S. sample showed that $95 \%$ of patients initiate on monotherapy in a time period before recent clinical trials and guideline updates that have shown benefits of combination therapy.

1 ulmonary arterial hypertension (PAH) is a condition characterized by elevated pulmonary arterial pressure due to vasoconstriction and remodeling of the vascular walls. ${ }^{1-3}$ PAH is often diagnosed in late stages and prognosis is poor, including right heart failure and premature death. ${ }^{1-3}$

Currently available targeted PAH-specific therapies comprise 4 medication classes-endothelin receptor agonists (ERAs), phosphodiesterase-5 inhibitors (PDE-5Is), prostacyclins, and soluble guanylate cyclase (sGC) stimulators-that aim to reduce vasoconstriction or inhibit abnormal cellular proliferation. ${ }^{2}$ Combination therapy, most commonly including 
an ERA and a PDE-5I, is increasingly common and may soon become the standard of care because PAH has several disease pathways and each medication class targets a different pathway. ${ }^{3}$ Until 2015, most guidelines advocated so-called sequential combination therapy, a single PAH-specific therapy at diagnosis with subsequent escalation to combination therapy as warranted after clinical re-evaluation. Recommendations for treatment have evolved with the advent of evidence in support of initial combination therapy, and the most recently published guidelines incorporate a class IB recommendation (i.e., recommendation that treatment is beneficial based on evidence from a single randomized trial) for initial ambrisentan (an ERA) and tadalafil (a PDE-5I) in treatment-naive patients whose symptoms are consistent with World Health Organization functional class (WHO-FC) II or III, which corresponds to patients with slight to marked limitations in physical activity. ${ }^{4,5}$ The use of initial combination therapy in these patients is consistent with treatment of other critical illnesses such as cancer and heart failure.

The recommendation is largely based on findings from the AMBITION trial, which demonstrated significant clinical benefit of the upfront combination of ambrisentan and tadalafil compared with initial therapy with either of these agents. ${ }^{6}$ There was a 50\% reduction in the primary endpoint of clinical failure (defined as death, hospitalization for worsening $\mathrm{PAH}$, disease progression, or unsatisfactory long-term clinical response) for patients treated with the upfront combination compared to the pooled monotherapy patients (ambrisentan monotherapy arm plus tadalafil monotherapy arm). From a health care resource perspective, hospitalization for worsening $\mathrm{PAH}$ was the clinical failure component with the greatest difference between treatment arms: $4 \%$ of combination therapy patients were hospitalized compared with $12 \%$ of pooled monotherapy patients.

The changing landscape of treatment options and recommendations may have a significant effect on health care resource utilization and costs. Treatment-related effect is not well reported in the literature, particularly regarding the balance of PAH-related health care utilization and associated costs. Pharmacy costs for PAH medications are high; therefore, medical costs must be reduced, presumably due to treatment effect, for use of these medications to reduce overall total medical costs. ${ }^{7}$ Hospitalization is common among PAH patients and represents a hard, real-world outcome to investigate due to its large effect on costs and its association with mortality. ${ }^{8-12}$ The high cost of PAH medications may be offset by cost savings from reductions in hospitalizations. ${ }^{13}$

In anticipation of the changing treatment patterns that may more often include combination therapy, we sought to examine the health care economic effect of PAH therapy prior to AMBITION and the guideline recommendation for upfront combination therapy. The current study examined claims data prior to 2015, characterized treatment patterns in the 6 months following treatment initiation, and evaluated health care resource utilization and costs of patients in the 6 months before and after treatment initiation. The primary aim was to compare overall cost before and after treatment, with an additional analysis of the various components including pharmacy and inpatient utilization.

\section{Methods}

\section{Study Design}

A retrospective, observational cohort design was used to describe PAH patients identified in an administrative claims database. An index date was assigned to each patient corresponding to the first observed date of an outpatient pharmacy claim for an approved PAH-specific medication between January 1, 2010, and December 31, 2014. The 6 months immediately preceding the index date were used to measure baseline patient characteristics, utilization, and costs. A 6-month follow-up period immediately after the index date was used to measure treatment patterns, utilization, and cost outcomes.

\section{Data Sources}

This study utilized U.S. insurance claims data from the Truven Health MarketScan Commercial Claims and Encounters and Medicare Supplemental and Coordination of Benefits Databases. These databases contain administrative claims data for approximately 80 million covered lives in 2010-2014 from approximately 150 large employers and health plans across the United States. Claims are fully adjudicated and represent the full continuum of care across all settings of care. Diagnosis, drug, and procedure codes on claims are used to define patient characteristics. The database represents claims from multiple, geographically diverse employer groups and health plans, which is a more representative sample of real-world data than claims from a single health plan or health care system.

The study databases comply with Sections 164.514 (a)-(b)lii of the Health Insurance Portability and Accountability Act of 1996 privacy rule regarding the determination and documentation of statistically deidentified data. Because the data are deidentified, institutional review board approval was not required prior to conducting the analysis.

\section{Study Sample Selection}

We identified patients with $\geq 1$ outpatient pharmacy claim with a National Drug Code (NDC) number or outpatient procedure with a Healthcare Common Procedure Coding System (HCPCS) code for any PAH treatment in the following therapeutic classes of interest: ERAs, PDE-5Is, or sGC stimulators. We explicitly excluded patients who began therapy with prostacyclin-class medications, as the clinical investigators felt that this small group of PAH patients would likely have very different patterns of health care utilization than those beginning typical oral PAH-specific therapy. To establish that these were newly treated patients, we required a clean period of at 


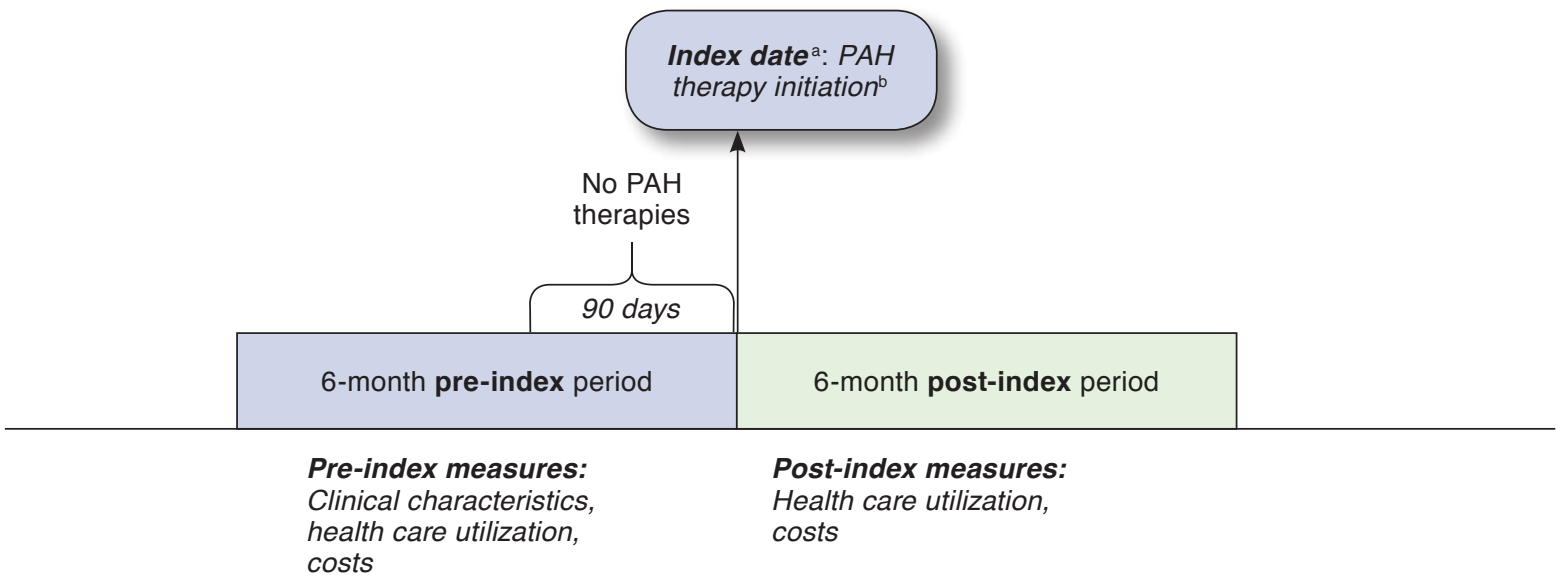

andex date is included in the post-index period.

Index date PAH therapies: ERAs, PDE-5Is, and SGC stimulators, in combination or monotherapy.

$E R A=$ endothelin receptor antagonist; $P A H=$ pulmonary arterial hypertension; $P D E-5 I=$ phosphodiesterase -5 inhibitor; $s G C=$ soluble guanylate cyclase

least 90 days with no claims for any PAH therapies, including prostacyclins, before the index date. We did include prostacyclin utilization and treatment patterns in the follow-up period. Patients were also required to have at least 6 months of continuous medical and prescription coverage prior to the index date and following the index date (Figure 1) and be at least 18 years of age on the index date.

Finally, patients were included if they had evidence of PAH, defined as meeting either criterion: (a) had at least 2 medical claims with a PAH diagnosis (International Classification of Diseases, 9th Revision, Clinical Modification [ICD-9-CM] diagnosis codes 416.0 and 416.8) in any position, at any time in the study period; or (b) had at least 2 medical claims with a PAH-related condition diagnosis (portal hypertension, connective tissue diseases, congenital heart disease, or human immunodeficiency virus) in any position at any time in the study period. Inclusion of PAH-related conditions in patient selection has been used in previous administrative claims-based studies of $\mathrm{PAH}$ due to the undercoding of $\mathrm{PAH} .^{13}$

As a subanalysis, we also stratified the PAH patient sample by those with and without claims for at least 1 previous right heart catheterization (RHC) during the 6-month baseline period. This procedure is performed to measure pulmonary artery pressure and diagnose $\mathrm{PAH}$.

\section{Treatment Pattern Outcomes}

PAH medication classes initiated on the index date through the first 30 days post-index were defined as the index regimen. Patients whose index regimen contained only 1 class were defined as initial monotherapy. Patients who received multiple classes in the first 30 days were classified as initial combination therapy. Although not considered a potential indexing medication class for monotherapy, the use of prostacyclins was considered a PAH treatment class if used in combination at index.

Over the 6-month post-index period, pharmacy and outpatient procedure claims corresponding to any PAH medication class not part of the index regimen were defined as sequential combination. The time from index date to sequential combination, and the class added, was described. Treatment interruption was defined as a gap lasting at least 30 days with no medication on hand, as indicated by fill dates and days' supply on pharmacy claims for the index regimen classes. Time to first treatment interruption and treatment restarts also were measured.

Treatment adherence was measured via medication possession ratio (MPR). MPR is defined as the proportion of follow-up days prior to an interruption (for patients with an interruption) or end of follow-up where the index regimen classes were on hand based on the dates and days supply provided on the applicable pharmacy claims, allowing patients to stockpile any overlapping prescriptions from early refills. Medical claims containing HCPCS codes for the administration of any medications were not included in the analysis as there is no assigned days supply. The MPR ranges from 0.0 to 1.0 , where 1.0 indicates that there were no missed days without drug on hand before either an interruption or the end of the 6-month followup. Typically, a threshold of 0.8 , or $80 \%$ of days with the drug on hand, is used to define a patient as adherent.

$$
\begin{gathered}
\text { MPR = total days of supply of index PAH medication } \\
\text { class / [total days from index date to end of 6-month } \\
\text { follow-up or treatment interruption] }
\end{gathered}
$$




\begin{tabular}{|c|c|c|}
\hline \multirow[b]{2}{*}{ Age in years (mean, SD) } & \multicolumn{2}{|c|}{$\begin{array}{l}\text { PAH Patients } \\
\mathrm{N}=3,908\end{array}$} \\
\hline & 62.7 & 14.7 \\
\hline Female (n, \%) & 2,473 & 63.3 \\
\hline \multicolumn{3}{|l|}{ Index year $(\mathrm{n}, \%)$} \\
\hline 2010 & 539 & 13.8 \\
\hline 2011 & 1,145 & 29.3 \\
\hline 2012 & 1,032 & 26.4 \\
\hline 2013 & 948 & 24.3 \\
\hline 2014 & 244 & 6.2 \\
\hline Deyo Charlson Comorbidity Index (mean, SD) & 2.5 & 2.1 \\
\hline \multicolumn{3}{|l|}{ Comorbid conditions present in baseline period (n, \%) } \\
\hline Other cardiovascular disease & 3,587 & 91.8 \\
\hline Diseases of the arteries, arterioles, and capillaries & 1,630 & 41.7 \\
\hline Atrial fibrillation & 984 & 25.2 \\
\hline Hypertension & 2,338 & 59.8 \\
\hline Chronic obstructive pulmonary disease & 1,422 & 36.4 \\
\hline Disorders of lipid metabolism & 1,208 & 30.9 \\
\hline Sleep apnea & 1,177 & 30.1 \\
\hline Type 2 diabetes & 1,120 & 28.7 \\
\hline Gastrointestinal disorders & 996 & 25.5 \\
\hline Upper gastrointestinal disorders & 562 & 14.4 \\
\hline Hepatic cirrhosis & 147 & 3.8 \\
\hline Mental disorder & 795 & 20.3 \\
\hline Dysthymia & 39 & 1.0 \\
\hline Connective tissue diseases & 735 & 18.8 \\
\hline Nontraumatic joint disorders & 619 & 15.8 \\
\hline Thyroid disease & 561 & 14.4 \\
\hline Diseases of the urinary system & 522 & 13.4 \\
\hline Overweight/obesity & 501 & 12.8 \\
\hline Anemia & 365 & 9.3 \\
\hline Acquired or hereditary hemolytic anemia & 40 & 1.0 \\
\hline Congenital heart disease & 285 & 7.3 \\
\hline Human immunodeficiency virus & 60 & 1.5 \\
\hline Schistosomiasis & 0 & 0.0 \\
\hline $\begin{array}{l}\text { Patients with at least } 3 \text { of the comorbidities listed } \\
\text { above (n, \%) }\end{array}$ & 3,205 & 82.0 \\
\hline \multicolumn{3}{|l|}{ Supportive therapy in baseline period (n, \%) } \\
\hline Diuretics & 2,579 & 66.0 \\
\hline Spironolactone & 597 & 15.3 \\
\hline Calcium-channel blockers & 1,500 & 38.4 \\
\hline Oxygen therapy & 1,182 & 30.2 \\
\hline Anticoagulants & 1,156 & 29.6 \\
\hline Digoxin & 451 & 11.5 \\
\hline \multicolumn{3}{|l|}{ Surgical procedures in baseline period (n, \%) } \\
\hline Atrial septostomy & 2 & 0.1 \\
\hline Lung transplant & 4 & 0.1 \\
\hline Heart-lung transplant & 1 & 0.0 \\
\hline Liver transplant & 4 & 0.1 \\
\hline Any of the above procedures & 11 & 0.3 \\
\hline$\underline{\text { RHC in baseline period (n, \%) }}$ & 2,158 & 55.2 \\
\hline \multicolumn{3}{|c|}{$\begin{array}{l}\text { aDemographic characteristics were measured on the index date (date of first PAH } \\
\text { therapy claim). Baseline clinical characteristics were measured in the 6-month } \\
\text { pre-index period (excluding the index date). } \\
\text { PAH = pulmonary arterial hypertension; } R H C=\text { right heart catheterization; } \\
\text { SD=standard deviation. }\end{array}$} \\
\hline
\end{tabular}

\section{Health Care Resource Utilization and Cost Outcomes}

Health care costs were measured during the 6-month pre- and post-index periods and expressed in 2014 constant U.S. dollars. Cost measures were inflation-adjusted to 2014 using the medical care component of the Consumer Price Index. Costs were calculated using financial fields that sum to the gross payment amount on claims incurred during the time frames of interest. Health care utilization was summarized as the proportion of patients with at least 1 of the following events as indicated by the type of claim: inpatient admission, emergency department (ED) visit, or outpatient office visit. PAH-related utilization and cost outcomes were calculated using the subset of administrative claims containing a diagnosis code for PAH in any position on the claim and outpatient pharmacy claims for PAH therapies.

\section{Statistical Analyses}

Demographics and clinical characteristics were described for the overall cohort and also stratified by patients with and without claims for RHC in the baseline period. Bivariate statistics were used to compare patient characteristics between those with and without RHC. Chi-squared tests and Student's t-tests were used to test for statistical significance for distributions of categorical variables and continuous variables, respectively. For the health care utilization and cost outcomes, paired t-tests and McNemar's tests were used to determine if mean costs and sample proportions were the same in the pre-index period vs the post-index period. For all comparisons, the threshold for statistical significance was set to $P \leq 0.05$.

\section{Results}

\section{Study Sample Characteristics}

A total of 29,900 patients were identified in the databases as having received PAH treatment during the years 2010-2014. The final sample of 3,908 patients met all criteria for evidence of PAH treatment initiation and had 2 additional claims for either PAH or a PAH-related condition during the baseline period.

The average age at index was over 60 years old and the sample was mostly female (Table 1). Over $90 \%$ of the sample indexed prior to 2014, indicating much of the sample began treatment prior to publication of the AMBITION trial of initial combination therapy with ambrisentan and tadalafil ${ }^{6}$ or the most recent treatment guidelines. ${ }^{4,5}$ In contrast to published guidelines for diagnosis, ${ }^{4,5}$ just over half the cohort had RHC. In general, demographic characteristics of those with and without RHC in the 6-month baseline period did not differ.

The clinical burden of PAH in this sample was compounded by the high prevalence of many of the comorbidities analyzed (Table 1). The most common comorbidities included other cardiovascular diseases, hypertension, chronic obstructive pulmonary disease, and disorders of lipid metabolism. Other supportive pharmacological therapies, such as diuretics, were 


\section{TABLE 2 Treatment Patterns}

\begin{tabular}{|c|c|c|}
\hline Index Therapy Characteristics ${ }^{a}$ & \multicolumn{2}{|c|}{$\begin{array}{l}\text { PAH Patients } \\
\mathrm{N}=3,908\end{array}$} \\
\hline \multicolumn{3}{|l|}{ Index therapy medications (n, \%) } \\
\hline ERAs & 993 & 25.4 \\
\hline Ambrisentan & 392 & 10.0 \\
\hline PDE-5Is & 3,042 & 77.8 \\
\hline Tadalafil & 896 & 22.9 \\
\hline sGC stimulators & 26 & 0.7 \\
\hline Initiated monotherapy (n, \%) & 3,715 & 95.1 \\
\hline Initiated combination therapy $\mathrm{b}(\mathrm{n}, \%)$ & 193 & 4.9 \\
\hline \multicolumn{3}{|l|}{ Treatment patterns } \\
\hline $\begin{array}{l}\text { Sequential combination (addition of second drug } \\
\text { class; } \mathbf{n}, \% \text { ) }\end{array}$ & 185 & 4.7 \\
\hline Time to sequential combination, in days (mean, SD) & 82.7 & 32.8 \\
\hline \multicolumn{3}{|c|}{$\begin{array}{l}\text { Sequential combination drug class added (of those with sequential } \\
\text { combination) (n, \%) }\end{array}$} \\
\hline ERAs & 88 & 47.6 \\
\hline PDE-5Is & 52 & 28.1 \\
\hline sGC stimulators & 1 & 0.5 \\
\hline Prostacyclins & 44 & 23.8 \\
\hline Treatment interruption (n, \%) & 1,489 & 38.1 \\
\hline Time to interruption, in days (mean, SD) & 59.6 & 36.0 \\
\hline $\begin{array}{l}\text { Restart therapy after interruption (of those with } \\
\text { interruption; } n, \% \text { ) }\end{array}$ & 497 & 33.3 \\
\hline \multicolumn{3}{|l|}{ Medication possession ratio (mean, SD) } \\
\hline ERAs & 0.954 & 0.112 \\
\hline PDE-5Is & 0.957 & 0.081 \\
\hline sGC stimulators & 0.958 & 0.065 \\
\hline Prostacyclins & 0.894 & 0.130 \\
\hline \multicolumn{3}{|c|}{$\begin{array}{l}\text { Index therapy medications were measured on index date and the following } 30 \text { days } \\
\text { bCombination therapy: more than } 1 \text { medication class added in the first } 30 \text { days } \\
\text { from index date. } \\
\text { ERA = endothelin receptor antagonist; } P A H=\text { pulmonary arterial hypertension; } \\
\text { PDE-5I = phosphodiesterase-5 inhibitor; } S D=\text { standard deviation; } S G C=\text { soluble } \\
\text { guanylate cyclase. }\end{array}$} \\
\hline
\end{tabular}

commonly used in the 6 months prior to initiating PAH therapy. Nearly one third of patients utilized oxygen therapy in the pre-index period. The average Deyo Charlson Comorbidity Index score $\mathrm{r}^{14}$ for the overall sample was 2.5.

\section{Treatment Patterns}

Nearly all patients initiated on monotherapy (Table 2). The most commonly prescribed medication class, either as monotherapy or as part of a combination regimen, was PDE-5Is, followed by ERAs. During the 6-month follow-up, nearly two fifths of patients experienced a treatment interruption of $>30$ days, and approximately one third of those patients restarted therapy.

\section{Health Care Resource Utilization and Costs}

The proportion of patients with at least 1 inpatient admission decreased significantly following treatment initiation (Figure 2). Likewise, PAH-related inpatient admissions fell by over 50\%
(7\%-3\%; $P<0.001)$. In contrast, while the proportion of patients with ED visits for any reason decreased (37\%-32\%; $P<0.001)$, the proportion of patients with a PAH-related ED visit increased (4\%-5\%; $P=0.014)$.

Nonpharmacy all-cause medical costs significantly decreased after PAH treatment initiation $(\$ 48,200$ [standard deviation \{SD\}: $\$ 117,686$ ] to $\$ 33,962$ [SD: $\$ 90,294$ ]; $P<0.001$ ), primarily driven by the reduction in inpatient costs $(\$ 32,322$ [SD: $\$ 110,993$ ] to $\$ 18,531$ [SD: $\$ 79,790]$; $P<0.001$; Figure 3). However, when including pharmacy costs, which increased significantly from $\$ 3,255$ (SD: $\$ 6,086$ ) to $\$ 19,961$ (SD: $\$ 18,720$ ), all-cause total costs remained steady after treatment initiation (\$51,455 [SD: $\$ 117,899]$ to $\$ 53,923$ [SD: $\$ 92,282] ; P=0.213)$.

Although all-cause spending remained stable before and after treatment initiation, PAH-related total costs increased significantly in the 6-month post-index period. Conversely, PAHrelated inpatient admission and nonpharmacy medical costs decreased significantly following treatment initiation, matching the trend of decreasing PAH-related inpatient admissions (Figure 3). All-cause total health care expenditures were higher among those with RHC in the baseline period compared with those without in both the pre-index and post-index periods. All-cause and PAH-related ED costs were not significantly different in the pre- and post-index periods.

\section{Discussion}

The study described a large sample of patients taking PAHspecific medications from a nationally representative administrative claims database in terms of patient characteristics, treatment patterns, and health care utilization and costs at the time of treatment initiation. Our study found that, on average, total all-cause health care spending did not differ significantly from the 6-month pre-index to post-index periods, where the index date represented the initiation of treatment with ERAs, PDE-5Is, and/or sGC stimulators. Results indicated that the increase in pharmacy costs after treatment initiation was balanced by a reduction in medical costs.

In addition to describing the effect of treatment initiation on utilization and costs, this study also provides an updated view of initial treatment choices in a real-world setting from 2010-2014. We found that approximately $95 \%$ of patients initiated monotherapy rather than combination treatment during this time. Many of the more recent drug efficacy studies permitted background therapy, and the 2015 European guidelines support a high-level recommendation (IB) for upfront combination therapy with ambrisentan and tadalafil as examples of the evolving treatment landscape, which may affect the proportion of patients initiating monotherapy in years 2015 and beyond.

Another important finding of this study is that over a third of patients had a treatment interruption lasting at least 30 days within the first 6 months of treatment initiation. This observation is similar to results from Sikirica et al. (2014), which 


\section{FIGURE 2 All-Cause and PAH-Related Health Care} Utilization $^{\mathrm{a}}$

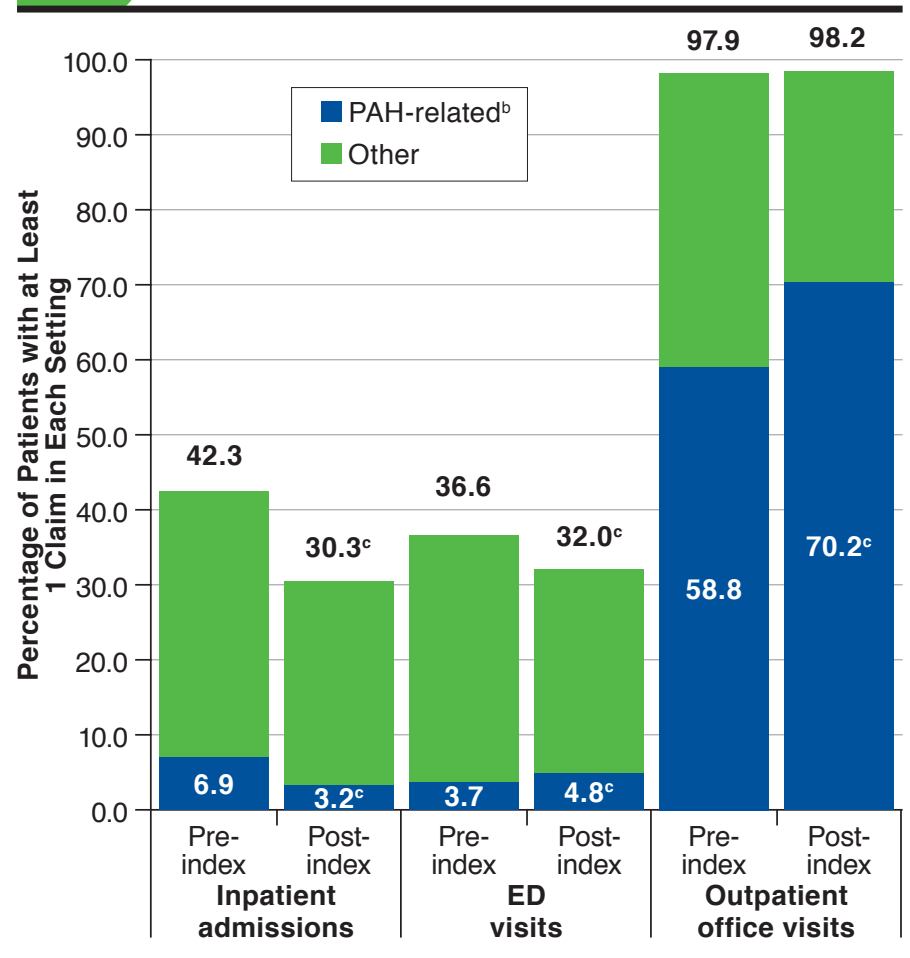

aUtilization was measured during 6-month pre-and post-index time periods; $N=3,908$.

bClaims considered PAH-related: inpatient admissions with a primary diagnosis of PAH; outpatient claims with a PAH diagnosis in any position; and claims for any of the index medications or prostacyclins.

cStatistically significant difference in pre-vs. post-index periods at $\alpha=0.05$ $P$ values were derived by the statistical comparison of pre-index and post-index proportions (McNemar's test).

$E D=$ emergency department; $P A H=$ pulmonary arterial hypertension.

showed that $38 \%$ of patients had a $\geq 60$-day gap in therapy (which more likely represents a true therapy interruption). ${ }^{13}$ Sikirica et al. noted that caps on pharmacy expenditures existed for some of the patients in their analysis. ${ }^{13}$ Filled prescriptions beyond the expenditure cap did not appear in the claims data they used for their analysis and may have affected the observed treatment interruptions. In addition, the claims data used by Sikirica et al., like the data used for the current analysis, could not account for patients having extra drug on hand because of previous early fills, a hospitalization, or samples-factors that may have made patients appear to have a treatment interruption when, in reality, there was no interruption-nor the exact reason behind any actual treatment interruption. ${ }^{13}$

Despite treatment interruptions, MPR rates for index regimens (approximately 95\% for each class) suggest patients were generally persistent with therapy over time. The MPR rates in this analysis were similar to previously reported 96\%-97\% rates in Copher et al. (2012). ${ }^{9}$

Our study confirms that a majority of PAH patients initiated on monotherapy in 2010-2014. A retrospective claims-based analysis by Copher et al. using data from 2004-2008 reported that $6 \%$ of patients initiated combination therapy when considering all classes prescribed in the first 90 days. ${ }^{9}$ Despite the longer time frame defining "initial combination," their result is similar to our observed rate of 5\% initial combination therapy. Angalakuditi et al. (2012) also used a 90-day window for defining the initial regimen; however, calcium-channel blockers were included as a PAH-specific treatment. ${ }^{15}$ Inclusion of this common, though unapproved, $\mathrm{PAH}$ therapy likely explains the higher proportion (18\%) considered to have started initial combination therapy. A cross-sectional analysis of all patients with PAH treatment in the Ontario Drug Benefit Program database identified 22\% treated with combination therapy, but this study's very broad definition of combination treatment as overlapping utilization of 2 or more drug classes in the years 2011-2012 almost certainly included many patients who had progressed to sequential combination therapy. ${ }^{16}$

Our study adds to the literature by analyzing the effect of treatment initiation on total patient health care costs in a pre-/ postperiod analysis using the latest available real-world data. Sikirica et al. showed a significant reduction in total costs (-16\%) when comparing the 12 months prior to treatment initiation with the 12 months post-initiation. ${ }^{13}$ Our study did not detect a significant change in total costs when analyzing 6-month periods of time, indicating that overall cost savings may be realized only after additional time for the treatment to improve symptoms and reduce overall health care utilization. Similar to the Sikirica et al. analysis, we observed lower medical costs offset by higher pharmacy costs after treatment initiation. ${ }^{13}$

Hospitalizations are often measured as an outcome in PAH due to the high cost of inpatient stays and the frequency at which PAH patients are hospitalized compared to the general population. The 8-year, 5,000 U.S. patient Registry to Evaluate Early and Long-term PAH Disease Management (REVEAL registry) found that $56.8 \%$ of participants were hospitalized over 3 years, with approximately half of the admissions (52.4\%) deemed PAHrelated based on the reason for hospitalization and primary discharge diagnosis available in the data. ${ }^{8}$ Our study also reported a high prevalence of hospitalizations, with 1 in 3 patients being admitted in the first 6 months after treatment initiation. Comparatively, our estimate was lower than that reported by Copher et al., but that study required patients to have an inpatient stay for PAH or an RHC procedure in the 90 days before treatment initiation while our study did not and may well have selected a population with higher resource utilization. ${ }^{9}$

Given the high prevalence of hospitalizations, the costs for hospitalizations are a significant contributor to the economic burden of PAH. Burke et al. (2015) reported that 
hospitalizations containing a primary or secondary diagnosis code for PAH among 1,543 commercially insured PAH patients had an average total cost per episode of $\$ 46,118$ (2011 USD). ${ }^{12}$ Copher et al. reported that per-patient per-month inpatient admission costs in the 6 months following treatment initiation were $\$ 3,701$ (2008 USD). ${ }^{9}$ Our results are in alignment with the Copher et al. estimate, with 6-month total inpatient costs averaging \$18,531 in the first 6 months, although our proportion of patients with at least 1 admission was lower. ${ }^{9}$ Anand et al. (2016) found that the absolute number of PAH-related hospitalizations in the United States decreased from 2001-2012, while average cost and length of stay increased. ${ }^{10}$ These results emphasize the need for continuing research on strategies to reduce the inpatient cost burden in PAH. We believe our larger, contemporary U.S. sample clearly establishes the economic burden of hospitalization in PAH patients and suggests that even large pharmacy expenses may well be offset by savings in health care utilization. The recently completed AMBITION study suggested initial combination therapy with ambrisentan and tadalafil would provide a superior reduction in PAH-related hospitalizations, ${ }^{6}$ and a similar, future cost analysis among patients who initiate combination therapy would be intriguing.

\section{Limitations}

The limitations of study design and generalizability require additional discussion. The data source for this study was an administrative claims database. Identifying $\mathrm{PAH}$ patients based on the presence of ICD-9-CM diagnosis codes recorded on medical claims or the receipt of $\mathrm{PAH}$-specific medication classes may be imprecise. We allowed patients to qualify for inclusion if they had at least 2 diagnoses for a PAH-related condition in the absence of a claim listing a specific PAH diagnosis given that the PAH-specific medication criterion would offer adequate specificity. This was similar to the approach used in Sikirica et al. ${ }^{13}$

Our results may underestimate the true cost of PAH-related events, because PAH may be undercoded. In addition, a single claim may underrepresent total resource utilization, but this methodology was employed due to the short time window of the study. We minimized this limitation by focusing on total health care costs, not just PAH-related costs.

RHC is the required diagnostic procedure for confirming the presence and severity of $\mathrm{PAH},{ }^{4,5}$ and the presence of a $\mathrm{PAH}$ related ICD-9-CM code does not necessarily indicate a diagnosis of the 5th World Symposium on Pulmonary Hypertension diagnostic group 1 PAH. ${ }^{17}$ Just over half of our sample had RHC claims in the pre-index period. The low rate is comparable to other published results but suggests the likelihood of diagnostic inaccuracy. ${ }^{18}$ To examine the potential effect, patients with and without RHC were analyzed. Those with RHC had higher rates of baseline comorbidities and higher costs in the preindex and post-index periods compared with those without

\section{FIGURE 3 All-Cause and PAH-Related Health} Care Costs $^{\mathrm{a}}$

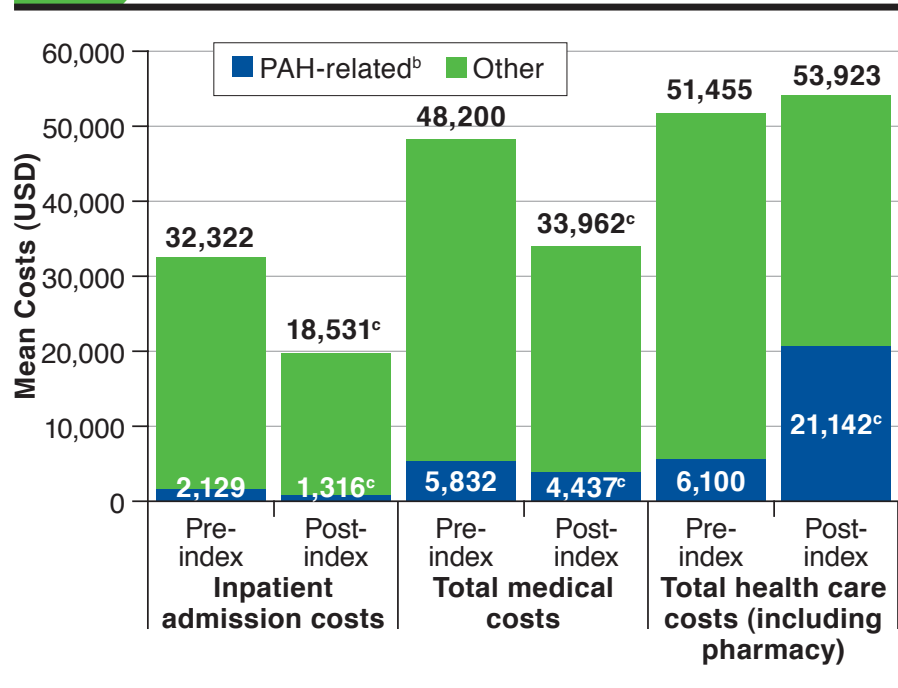

aCosts were summed during 6 month pre- and post-index time periods; $N=3,908$. ${ }^{b}$ Claims considered PAH-related: inpatient admissions with a primary diagnosis of PAH; outpatient claims with a PAH diagnosis in any position; and claims for any of the index medications or prostacyclins.

cStatistically significant difference in pre-vs. post-index periods at $\alpha=0.05$.

$P$ values were derived by the statistical comparison of pre-index and post-index mean costs (paired t-test).

$P A H=$ pulmonary arterial hypertension .

RHC. It is possible patients with RHC may have been more likely to have true group $1 \mathrm{PAH}$ that is associated with worse clinical health prior to treatment initiation, thereby increasing costs. The RHC procedure itself substantially affects pre-index costs, and post-index cost differences may be attributable to factors other than severity of illness. Due to the large range of potential confounders, a more in-depth comparison was not preformed. Future health care cost studies should consider the impact of including RHC as an inclusion criteria or cost category when describing this disease area.

The patient selection criteria utilized in our study may limit comparability to previously reported PAH-related cost estimates, but we believe our strategy is a strength that increases the generalizability of our data. We speculate that requiring a PAH diagnosis during the baseline period generates a population in which PAH diagnoses are more likely to appear on subsequent claims, thus driving PAH-related utilization post-index. For example, our study showed PAH-related costs were a small proportion of total costs, but Copher et al., which required a PAH-related inpatient admission in the baseline period for study inclusion, showed PAH-related costs comprised $71 \%$ of all-cause costs in the post-index period. ${ }^{9}$ Moreover, $<5 \%$ of patients in our study had a PAH-related inpatient admission in the post-index period, while $55 \%$ of patients in Copher et al. 


\section{Treatment Patterns and Associated Health Care Costs Before and After Treatment Initiation Among Pulmonary Arterial Hypertension Patients in the United States}

had such an admission. ${ }^{9}$ Sikirica et al. utilized a different selection process that provided the basis for patient selection in our study and which we speculate identified a more representative group of PAH patients. ${ }^{13}$

In this real-world claims dataset, we almost certainly included patients other than the WHO Group 1 PAH patients for whom the drugs are approved. Compared to patients in the REVEAL study, ${ }^{8}$ which was limited to WHO Group 1 PAH patients based on confirmatory RHC and managed at expert centers, patients in this study were approximately 10 years older (53 vs. 62.7 years old), with a lower proportion of females (80\% vs. $63.3 \%$ ) and fewer comorbid conditions. These differences suggest inclusion of non-group $1 \mathrm{PAH}$ patients in our study; however, other possible explanations include an age bias in referral or treatment approach, practice differences, and/or patient preference in a real-world setting as opposed to the PAH referral centers that enrolled the REVEAL cohort.

\section{Conclusions}

This study provides much needed real-world information regarding treatment patterns and patient health care utilization and costs during a time of evolving treatment options and guidelines. In our large, representative U.S. sample, monotherapy initiation was far more common than combination therapy just prior to recent years' growing clinical trial evidence supporting initial combination therapy for PAH patients. Treatment initiation was found to have no significant effect on total health care spending because, while PAH-related pharmacy costs increased after treatment initiation, the increase was offset by reduced inpatient utilization. Most patients in this study were treated with monotherapy, but because the AMBITION study ${ }^{15}$ indicated initial combination therapy with ambrisentan and tadalafil reduced PAH-related hospitalizations compared to initial monotherapy with these agents, future studies should compare health care utilization and total costs for patients who begin these treatments. Such an analysis would add real-world pharmacoeconomic evidence to complement recent clinical trial findings and monitor the clinical and economic impacts of evolving PAH management strategies.

\section{Authors}

CHARLES D. BURGER, MD, FCCP, Mayo Clinic, Jacksonville, Florida, and R. JAMES WHITE, MD, PhD, University of Rochester Medical Center; Rochester, New York. A. BURAK OZBAY, MBA, $P h D$, and HOWARD M. LAZARUS, MD, Gilead Sciences, Foster City, California. ELLEN RIEHLE, MPH; LESLIE B. MONTEJANO, MA, CCRP; and GREGORY LENHART, MS, Truven Health Analytics, an IBM company, Ann Arbor, Michigan.

AUTHOR CORRESPONDENCE: Charles D. Burger, MD, FCCP, Mayo Clinic, 4500 San Pablo Rd., S., Jacksonville, FL 32224

Tel.:904.953.2869; E-mail: burger.charles@mayo.edu.

\section{DISCLOSURES}

This study was sponsored and funded by Gilead Sciences. Ozbay is an employee of Gilead Sciences. At the time that this project and manuscript were developed, Lazarus was an employee of Gilead Sciences and may own stock/stock options. Riehle, Montejano, and Lenhart are employees of Truven Health Analytics, an IBM company, which received funding from Gilead Sciences to conduct this study. Burger and White do research with, and are paid consultants for, Gilead Sciences; they do not own equity and received no personal compensation for the work here. Burger also reports consultancy and advisory board work for Actelion Pharmaceuticals and grants from Gilead Sciences, Actelion Pharmaceuticals, Bayer, and United Therapeutics.

\section{ACKNOWLEDGMENTS}

Programming support for this analysis was provided by Helen Varker and Diana Stetsovsky of Truven Health Analytics, an IBM company.

\section{REFERENCES}

1. Corris P, Degano B. Severe pulmonary arterial hypertension: treatment options and the bridge to transplantation. Eur Respir Rev. 2014;23(134):488-97.

2. Seferian A, Simonneau G. Therapies for pulmonary arterial hypertension: where are we today, where do we go tomorrow? Eur Respir Rev. 2013;22(129):217-26.

3. Ghofrani HA, Humbert M. The role of combination therapy in managing pulmonary arterial hypertension. Eur Respir Rev. 2014;23(134):469-75.

4. Galiè N, Humbert M, Vachiery JL, et al. 2015 ESC/ERS guidelines for the diagnosis and treatment of pulmonary hypertension: the Joint Task Force for the Diagnosis and Treatment of Pulmonary Hypertension of the European Society of Cardiology (ESC) and the European Respiratory Society (ERS): Endorsed by: Association for European Paediatric and Congenital Cardiology (AEPC), International Society for Heart and Lung Transplantation (ISHLT). Eur Heart J. 2016;37(1):67-119.

5. Galiè N, Humbert M, Vachiery JL, et al. 2015 ESC/ERS guidelines for the diagnosis and treatment of pulmonary hypertension. Eur Respir J. 2015;46(4):903-75.

6. Galiè N, Barbera JA, Frost AE, et al. Initial use of ambrisentan plus tadalafil in pulmonary arterial hypertension. N Engl J Med. 2015;373:834-44

7. Selexipag (Uptravi) for pulmonary arterial hypertension. Med Lett Drugs Ther. 2016;58(1488):21-23

8. Burger CD, Long PK, Shah MR, et al. Characterization of first time hospitalizations in patients with newly diagnosed pulmonary arterial hypertension in the REVEAL registry. Chest. 2014;146(5):1263-73.

9. Copher R, Cerulli A, Watkins A, Laura Monsalvo M. Treatment patterns and healthcare system burden of managed care patients with suspected pulmonary arterial hypertension in the United States. J Med Econ. 2012;15(5):947-55.

10. Anand V, Roy SS, Archer SL, et al. Trends and outcomes of pulmonary arterial hypertension-related hospitalizations in the United States: analysis of the Nationwide Inpatient Sample database from 2001 through 2012. JAMA Cardiol. 2016;1(9):1021-29.

11. Frantz RP, Schilz RJ, Chakinala MM, et al. Hospitalization and survival in patients using epoprostenol for injection in the PROSPECT observational study. Chest. 2015;147(2):484-94.

12. Burke JP, Hunsche E, Regulier E, Nagao M, Buzinec P, Drake III W. Characterizing pulmonary hypertension-related hospitalization costs among Medicare Advantage or commercially insured patients with pulmonary arterial hypertension: a retrospective database study. Am J Manag Care. 2015;21(3 Suppl):s47-58

13. Sikirica M, Iorga SR, Bancroft T, Potash J. The economic burden of pulmonary arterial hypertension (PAH) in the U.S. on payers and patients. BMC Health Serv Res. 2014;14:676. 
14. Deyo RA, Cherkin DC, Ciol MA. Adapting a clinical comorbidity index for use with ICD-9-CM administrative databases. J Clin Epidemiol. 1992;45(6):613-19.

15. Angalakuditi M, Edgell E, Beardsworth A, Buysman E, Bancroft T. Treatment patterns and resource utilization and costs among patients with pulmonary arterial hypertension in the United States. J Med Econ. 2010;13(3):393-402

16. Vaid HM, Camacho X, Granton JT, et al. The characteristics of treated pulmonary arterial hypertension patients in Ontario. Can Respir J. 2016;2016:6279250

17. Simonneau G, Gatzoulis MA, Adatia I, et al. Updated clinical classification of pulmonary hypertension. J Am Coll Cardiol. 2013;62(25 Suppl):D34-41
18. Duarte AG, Lin YL, Sharma G. Incidence of right heart catheterization in patients initiated on pulmonary arterial hypertension therapies: a population-based study. J Heart Lung Transplant. 2016;36(2):20-226.

19. Mehta S, Sastry BK, Souza R, et al. Macitentan improves health-related quality of life for patients with pulmonary arterial hypertension: results from the randomized controlled SERAPHIN trial. Chest. 2017;151(1):106-18. 20. Galiè N, Grimminger F, Grünig E, et al. Comparison of hemodynamic parameters in treatment-naïve and pre-treated patients with pulmonary arterial hypertension in the randomized phase III PATENT-1 study. J Heart Lung Transplant. 2017;36(5):509-19. 